ARTIGO ORIGINAL

ISSN 1677-5090

(C) 2018 Revista de Ciências Médicas e Biológicas

DOI: http://dx.doi.org/10.9771/cmbio.v17i3.28673

\title{
Precisão de medidas de força muscular isométrica com dinamometria manual
}

\author{
Accuracy of isometric muscular force measures with manual dynamometry
}

\author{
Mariana Barcellos Machado Bertoni ${ }^{1}$, Mansueto Gomes Neto ${ }^{2 *}$
}

\begin{abstract}
${ }^{1}$ Mestranda em Processos Interativos de Órgãos e Sistemas pela Universidade Federal da Bahia; ${ }^{2}$ PhD, Professor Permanente do Programa de Pós-Graduação em Processos Interativos de Órgãos e Sistemas da Universidade
\end{abstract}

Federal da Bahia.

\begin{abstract}
Resumo
Objetivo: avaliar a precisão de medidas de força muscular isométrica máxima, realizadas através da dinamometria manual isométrica dos músculos responsáveis por movimentos do quadril. Metodologia: foi realizado um teste de força da musculatura do quadril em 10 voluntários saudáveis. Todos eles realizaram três repetições de força isométrica máxima, com intervalo de 15 segundos entre elas. A análise dos dados foi feita através do Coeficiente de Correlação Intraclasse (CCl) oneway, tipo consistência. Resultados: o coeficiente de correlação intraclasse (CCI), para todos os grupos musculares, ficou acima de 0,9 , o que significa resultados excelentes de concordância entre as medidas. Conclusão: para o teste de dinamometria manual descrito, resultados similares foram encontrados entre a primeira, a segunda e a terceira medida realizada. Dessa forma, podemos dizer que o avaliador é capacitado para a realização dessa avaliação. Além disso, a presença de resultados consistentes em todas as três medidas significa que qualquer uma delas poderá ser utilizada para análises estatísticas posteriores.

Palavras-chave: Força muscular. Dinamometria. Contração muscular.
\end{abstract}

\begin{abstract}
Objective: evaluate the accuracy of maximum isometric muscle strength measurements carried out by isometric manual dynamometry of muscles responsible for movement of the hip. Methodology: a test of strength of the muscles of the hip was conducted in 10 healthy volunteers. All of them performed 3 repetitions of maximum isometric strength, with an interval of 15 seconds between them. Data analysis was made by using the Intraclass Correlation Coefficient (ICC) one-way type consistency. Results: the intraclass correlation coefficient (ICC) for all muscle groups stayed above 0.9, which means excellent results of concordance between measures. Conclusion: for the described manual dynamometry test, similar results have been found among the first, second and third measure. In this way, we can say that the evaluator is able to carry out this evaluation. In addition, the presence of consistent results on all three measures means that any one of them could be used for subsequent statistical analyses.
\end{abstract}

Keywords: Muscle strength. Dynamometry. Muscle contraction.

\section{INTRODUÇÃO}

A força muscular tem um papel relevante na realização de diversas atividades da vida diária. Além de ser um importante componente na saúde e na aptidão física, é conhecida como importante preditor de função (HISLOP et al., 2014).

Avaliações de força muscular são frequentemente usadas no ambiente clínico para medir a performance muscular, permitindo ao fisioterapeuta diagnosticar fraquezas ou alterações, comparar eficácia de tratamentos e guiar possíveis intervenções (KOBLBAUER et al., 2011). O conhecimento da força muscular máxima que um indivíduo é capaz de produzir é um componente necessário para se elaborar um programa de reabilitação adequado (DANNESKIOLD-SAMS $\varnothing$ E et al., 2009).

Correspondente/Corresponding: * Mansueto Gomes Neto - End: Universidade Federal da Bahia - PAC - Depto de Fisioterapia - Tel: (71)99918-8277 - E-mail: netofisio@gmail.com
A dinamometria isocinética é considerada padrão de referência para a avaliação de desempenho muscular e, apesar de ser muito descrita na literatura, é pouco utilizada na prática clínica de fisioterapeutas, no esporte ou em academias, pela dificuldade de operacionalização, pelo alto custo e o tempo necessário (STARK et al., 2011). Dessa forma, é provável que o diagnóstico e a prescrição dos exercícios e programas de reabilitação sejam comprometidos.

A alternativa mais viável é a utilização da dinamometria manual isométrica, um método também amplamente descrito na literatura, de fácil manuseio e menor custo quando comparado ao padrão ouro (NIMS, 1996).

O uso de procedimentos padronizados para a avaliação da força muscular isométrica máxima através da dinamometria manual tem demonstrado boa confiabilidade e validade em crianças (BEENAKKER et al., 2001; ROSE et al., 2008), adultos (BURNS et al., 2005) e idosos (ARNOLD et al., 2010). É possível melhorar ainda mais a acurácia da avaliação utilizando a fixação do dinamô- 
metro, principalmente em grupos musculares maiores e capazes de gerar maior torque (MARTIN et al., 2006).

Além disso, destacamos a necessidade de identificação de valores normativos para tornar o método passível de comparação entre diferentes idades, sexo ou outros fatores relacionados (DANNESKIOLD-SAMS $\varnothing \mathrm{E}$ et al., 2009). Com base nisso, alguns estudos foram realizados buscando valores de referência na população. Dessa forma, torna-se possível aprimorar a elaboração do diagnóstico e a aplicação de exercícios na prática fisioterapêutica, bem como a prescrição de atividade física para indivíduos saudáveis (BENFICA et al., 2018; McKAY et al., 2017).

O presente estudo tem como objetivo analisar a concordância das medidas de contração isométrica máxima, obtidas através da dinamometria manual, por um avaliador treinado e familiarizado com o método.

\section{METODOLOGIA}

A amostra do estudo foi de conveniência, composta por voluntários saudáveis, caracterizados por: ausência de sintomas nociceptivos musculoesqueléticos nos últimos 2 meses; idade entre 18 e 80 anos; ter assinado o termo de consentimento livre e esclarecido (TCLE). Foram excluídos: indivíduos sem incapacidade cognitiva para compreender o protocolo e as orientações; pacientes com disfunções neuromusculares diagnosticadas; pacientes com redução de amplitude de movimento funcional; e pacientes com alguma condição cardiorrespiratória ou sistêmica que contraindicasse a realização do protocolo de avaliação da força muscular. Todos os voluntários incluídos na pesquisa foram submetidos a entrevista para levantamento de dados sociodemográficos e funcionais.

A avaliação da força muscular isométrica foi realizada utilizando-se o dinamômetro hand held, modelo 01165, da Marca Lafayette Instrument (Lafayette, Sagamore, USA). A avaliação foi realizada em local reservado e apropriado para a avaliação. Foi avaliada a contração isométrica bilateral dos grupos musculares da articulação do quadril. Previamente à mensuração, o voluntário foi orientado e treinado para cada movimento. Após a contração isométrica por três segundos, um bip sonoro do próprio equipamento, sinalizava o final da avaliação para cada movimento. Cada movimento foi realizado três vezes, com intervalo de 15 segundos. Para garantir a interferência mínima do examinador na medida final de força, foi utilizada uma faixa inelástica, com fixação através de um dispositivo de ventosa, ligada a um ponto fixo, ou manual quando o fixo não era possível, sempre com resistência máxima para anular o vetor de movimento e garantir que o torque mensurado era o maior associado à contração isométrica.
Foram avaliados os seguintes grupos musculares da articulação do quadril: flexores, extensores, abdutores, adutores, rotadores internos e rotadores externos. As posições do sujeito avaliado e do dinamômetro manual, adotadas durante a coleta, estão apresentadas na Tabela 1.

Previamente à realização do estudo, a pesquisa foi aprovada pelo comitê de ética em pesquisa do Instituto de Ciências da Saúde da Universidade Federal da Bahia, com parecer $n^{\circ} 1537948$. A pesquisa foi realizada com uma amostra de conveniência, e todos os voluntários assinaram o TCLE. A pesquisa garantiu a integridade e manteve em sigilo os dados individuais dos voluntários que compuseram a amostra, em consonância com as recomendações da Resolução 466/12 CNS/MS.

A análise dos dados foi feita por meio do Coeficiente de Correlação Intraclasse (CCI) oneway tipo consistência (intraobservador). O ICC varia de 0 a 1 , e quanto mais próximo de 1 maior a concordância intraobservador e melhor a consistência do examinador para realizar a avaliação descrita (ROSNER et al., 2006; VIEIRA, 2003).

Tabela 1 - Descrição do posicionamento durante a avaliação de força muscular isométrica.

\begin{tabular}{|c|c|c|c|}
\hline Grupo muscular & $\begin{array}{l}\text { Posição do } \\
\text { paciente }\end{array}$ & $\begin{array}{l}\text { Posição } \\
\text { articular }\end{array}$ & $\begin{array}{l}\text { Posição do } \\
\text { dinamômetro }\end{array}$ \\
\hline Flexores quadril & Decúbito dorsal & $\begin{array}{l}\text { Quadril fletido } \\
90^{\circ} \text { e joelho } \\
\text { relaxado }\end{array}$ & $\begin{array}{l}\text { Coxa distal, região } \\
\text { dorsal. }\end{array}$ \\
\hline Extensores quadril & Decúbito ventral & $\begin{array}{l}\text { Quadril } \\
\text { e joelho } \\
\text { estendidos }\end{array}$ & $\begin{array}{l}\text { Coxa distal, região } \\
\text { ventral. }\end{array}$ \\
\hline Abdutores quadril & Decúbito dorsal & $\begin{array}{l}\text { Quadril em } \\
\text { neutro }\end{array}$ & $\begin{array}{l}\text { Coxa distal, região } \\
\text { lateral. }\end{array}$ \\
\hline Adutores quadril & Decúbito ventral & $\begin{array}{l}\text { Quadril em } \\
\text { neutro }\end{array}$ & $\begin{array}{l}\text { Coxa distal, região } \\
\text { medial. }\end{array}$ \\
\hline $\begin{array}{l}\text { Rotadores internos } \\
\text { quadril }\end{array}$ & Sedestação & $\begin{array}{l}\text { Quadril e } \\
\text { joelho fletidos } \\
90^{\circ}\end{array}$ & $\begin{array}{l}\text { Próximo ao } \\
\text { maléolo lateral. }\end{array}$ \\
\hline $\begin{array}{l}\text { Rotadores externos } \\
\text { quadril }\end{array}$ & Sedestação & $\begin{array}{l}\text { Quadril e } \\
\text { joelho fletidos } \\
90^{\circ}\end{array}$ & $\begin{array}{l}\text { Próximo ao } \\
\text { maléolo medial. }\end{array}$ \\
\hline
\end{tabular}

Fonte: Dados da pesquisa.

\section{RESULTADOS}

A amostra foi composta por 10 voluntários, com idades entre 24 e 38 anos, idade média de 30 anos, com predomínio do sexo masculino (70\%), todos eles com lateralidade dominante à direita.

O coeficiente de correlação intraclasse $(\mathrm{CCl})$ para todos os grupos musculares ficou acima de 0,9 , o que significa resultados excelentes de concordância entre as medidas, com destaque para os rotadores internos de quadril, com $\mathrm{CCl} 0,995$ à direita e 0,994 à esquerda (Tabela 2). 
Tabela 2 - Valores do coeficiente de correlação intraclasse (CCl) e intervalo de confiança

\begin{tabular}{lcccc}
\hline Grupos musculares & & & & \\
Extensores de quadril & IC 95\% & \\
\hline Flexores de quadril & D & 0,99 & $(0,973-0,997)$ \\
& E & 0,966 & $(0,906-0,99)$ \\
\hline Abdução de quadril & D & 0,984 & $(0,956-0,996)$ \\
& E & 0,984 & $(0,955-0,996)$ \\
\hline Adução de quadril & D & 0,982 & $(0,951-0,995)$ \\
& E & 0,988 & $(0,967-0,997)$ \\
\hline Rotadores internos de quadril & D & 0,988 & $(0,965-0,997)$ \\
& E & 0,983 & $(0,954-0,995)$ \\
\hline Rotadores externos de quadril & E & 0,994 & $(0,986-0,999)$ \\
& D & 0,983 & $(0,983-0,998)$ \\
\hline
\end{tabular}

Fonte: Dados da pesquisa

\section{DISCUSSÃO}

Os resultados do presente estudo demonstraram que, para o teste de dinamometria manual descrito, resultados similares foram encontrados entre a primeira, a segunda e a terceira medida realizadas. Dessa forma, podemos dizer que o avaliador é capacitado para a realização da avaliação de força muscular isométrica, o que poderá ser utilizado em estudos futuros. Além disso, a presença de resultados consistentes em todas as três medidas significa que qualquer uma delas poderá ser utilizada para análises estatísticas posteriores.

O Coeficiente de Correlação Intraclasse (CCI) é indicado como índice mais adequado para a confiabilidade de análises quantitativas, e valores maiores ou iguais a 0.75 indicam confiabilidade aceitável (LEXELL; DOWNHAM, 2005). Os valores encontrados neste estudo estão todos acima de 0.9 , semelhantes aos que foram encontrado na avaliação de força muscular dos flexores plantares com fixação do dinamômetro (DAVIS et al., 2017). Podemos ressaltar que a padronização da coleta, realizada após extensa revisão da literatura, é um fator importante para a obtenção de resultados confiáveis e reprodutíveis.

É importante pontuar que a realização de uma medida, após a familiarização com o teste, não reduziu a confiabilidade do coeficiente intraclasse, o que poderia justificar a redução do número de medidas realizadas durante a avaliação de força muscular isométrica máxima. A maioria dos estudos publicados utiliza três medidas com intervalos que variam de 10 a 1 segundo (BACKMAN et al., 1995; HOGREL et al., 2007), mas alguns estudos mais recentes já utilizaram apenas duas medidas e apresentaram redução no tempo total de coleta (CHEZAR et al., 2013; WESTRICK et al., 2013).
No entanto, considerando a pesquisa científica, tal procedimento representaria uma diminuição de tempo e, consequentemente, de recursos despendidos em uma coleta com grande número de voluntários, por exemplo, em um estudo de valores normativos. Além disso, diminuiria o risco da fadiga à qual o voluntário é exposto durante a coleta. Já na prática clínica, poderia melhorar a viabilidade e a aplicabilidade do teste dentro de um conjunto de ferramentas utilizadas para avaliação de fisioterapeutas ou educadores físicos.

O uso de uma amostra pequena, por conveniência e não randomizada, é uma limitação do estudo pois não permitiu aprofundar a análise estatística. Estudos futuros devem continuar investigando a confiabilidade das medidas.

\section{CONCLUSÃO}

Para o teste de dinamometria manual descrito, resultados similares foram encontrados entre a primeira, a segunda e a terceira medida realizada. Dessa forma, podemos dizer que o avaliador é capacitado para a realização dessa avaliação. Além disso, a presença de resultados consistentes em todas as três medidas significa que qualquer uma delas poderá ser utilizada para análises estatísticas posteriores.

\section{REFERÊNCIAS}

ARNOLD, C. M. et al. The reliability and validity of handheld dynamometry for the measurement of lower-extremity muscle strength in older adults. J. strength. cond. res., Lincoln, v. 24, n. 3, p. 815-824, 2010.

BACKMAN, E. et al. Isometric muscle strength and muscular endurance in normal persons aged between 17 and 70 years. Scand. J. Rehab. Med., Stockholm, v. 27, n. 2, p. 109-117, 1995.

BENFICA, P. A. et al. Reference values for muscle strength: a systematic review with a descriptive meta-analysis. Braz. j. phy.s ther., São Carlos, v. 122 , p.1-15, 2018.

BEENAKKER, E. A. C. et al. Reference values of maximum isometric muscle force obtained in 270 children aged $4-16$ years by hand-held dynamometry. Neuromuscul. disord., Oxford, v. 11, n. 5, p. 441-446, 2001.

BURNS, J. et al. Quantification of muscle strength and imbalance in neurogenic pes cavus, compared to health controls, using hand-held dynamometry. Foot ankle int., Baltimore, v. 26, n. 7, p. 540-544, 2005.

CHEZAR, A. et al. Normal isometric strength of rotator cuff muscle in adults. Bone Joint Res., [s.I], v. 2, n. 10, p. 214-219, 2013.

DANNESKIOLD - SAMS $\varnothing \mathrm{E}$, B. et al. Isokinetic and isometric muscle strength in a healthy population with special reference to age and gender. Acta physiol., Oxford, v. 197, suppl. 673, p.1-68, 2009.

DAVIS, P. R. et al. Repeatability, consistency, and accuracy of hand-held dynamometry with and without fixation for measuring ankle plantarflexion strength in healthy adolescents and adults. Muscle Nerve, Boston, v. 56, n. 5, p. 896-900, 2017.

HISLOP, H. J. et al. Testing techniques of manual examination and performance testing. 9th ed. St. Louis: Elsevier Sanders, 2014.

HOGREL, J. Y. et al. Development of a French isometric strength normative database for adults using quantitative muscle testing. Arch. phys. 
med. rehabil., Chicago, v. 88, n. 10, p. 1289-1297, 2007.

KOBLBAUER, I. F. et al. Reliability of maximal isometric knee strength testing with modified hand-held dynamometry in patients awaiting total knee arthtoplasty: useful in research and individual patient settings? A reliability study. BMC musculoskelet. disord., London,n. 12, p. 249, 2011.

LEXELL, J.; DOWNHAM, D.Y. How to assess the reliability of measurements in rehabilitation. Am J Phys Med Rehabil, [s.I], v. 84, n. 9, p. 719-723, 2005.

MARTIN, H. J. et al. Is hand-held dynamometry useful for the measurement of quadríceps strength in older people? A comparision with the gold standard bodex dynamometry. Gerontology, Basel, v. 52, n. 3, p.154-159, 2006.

McKAY, M. J. et al. Normative reference values for strength and flexibility of 1.000 children and adults. Neurology, New York, v. 88, n. 1, p.
36-43, 2017.

ROSE, K. J. et al. Reliability of quantifying foot and ankle muscle strength in very young children. Muscle nerve, Boston , v. 37, n. 5, p. 626-631, 2008.

ROSNER, B. Fundamentals of biostatistics. Belmont, CA: Thomson-Brooks/Cole, 2006.

STARK, T. et al. Hand-held dynamometry correlation with the gold standard isokinetic dynamometry: a systematic review. Crit. Rev., [s.I], v. 3, n. 5, p. 472-479, 2011.

VIEIRA, S. Bioestatística: tópicos avançados. São Paulo: Campus, 2003.

WESTRICK, R. B. et al. Isometric shoulder strength reference values for physically active collegiate males and females. Sports Health, United States, v. 5, n. 1, p. 17-21, 2013.

Submetido em: 19/11/2018

Aceito em: 29/11/2018 